\title{
Site testing in summer at Dome C, Antarctica
}

\author{
E. Aristidi ${ }^{1}$, A. Agabi ${ }^{1}$, E. Fossat ${ }^{1}$, M. Azouit ${ }^{1}$, F. Martin ${ }^{1}$, T. Sadibekova ${ }^{1}$, T. Travouillon ${ }^{2}$, J. Vernin ${ }^{1}$, and A. Ziad ${ }^{1}$ \\ 1 Laboratoire Universitaire d'Astrophysique de Nice, Université de Nice Sophia Antipolis, Parc Valrose, \\ 06108 Nice Cedex 2, France \\ e-mail: aristidi@unice.fr \\ 2 School of Physics, University of New South Wales, Sydney, NSW 2052, Australia
}

Received 28 May 2005 / Accepted 23 August 2005

\section{ABSTRACT}

We present summer site testing results based on DIMM data obtained at Dome C, Antarctica. These data were collected on the bright star Canopus during two 3-months summer campaigns in 2003-2004 and 2004-2005. We performed continuous monitoring of the seeing and the isoplanatic angle in the visible. We found a median seeing of $0.54^{\prime \prime}$ and a median isoplanatic angle of $6.8^{\prime \prime}$. The seeing appears to have a deep minimum around $0.4^{\prime \prime}$ almost every day in late afternoon.

Key words. site testing

\section{Introduction}

The French (IPEV) and Italian (ENEA) polar Institutes have been constructing the Concordia base on the Dome $\mathrm{C}$ site of the Antarctic plateau $\left(75^{\circ} \mathrm{S}, 123^{\circ} \mathrm{E}\right)$ at an elevation of $3250 \mathrm{~m}$ that corresponds, given the cold air temperatures, to an air pressure encountered around $3800 \mathrm{~m}$ at more standard latitudes. The Concordia construction is now completed and the first winterover started in 2005. Astronomy is obviously near the top of the list of the scientific programmes that will benefit from this unique site: the extremely cold and dry air is complemented by very low winds, both at ground level and at higher altitude, so that an exceptionally good seeing is expected.

In the late 90's a site testing program based on balloonborne microthermal sensors was conducted by J. Vernin and R. Marks at South Pole (Marks et al. 1999). As katabatic winds are present at the South Pole, these authors found poor seeing from the ground (recent measurements by Travouillon et al. 2003 confirmed a value of $1.7^{\prime \prime}$ in the visible range). An amazing result is that the seeing drops down to $0.3^{\prime \prime}$ at $200 \mathrm{~m}$ height above the surface, i.e. at an altitude of $3050 \mathrm{~m}$ above sea level. Therefore Dome C, with its $3250 \mathrm{~m}$ altitude, in a low wind area, appeared an excellent candidate for astronomy.

These promising qualities encouraged our group to initiate a detailed analysis of the astronomical site properties. In 1995, a Franco-Italian group directed by J. Vernin made a pioneering prospective campaign at Dome $\mathrm{C}$ and launched a few meteorological balloons. A systematic site-testing programme was then initiated under the name of Concordiastro, first funded by IPEV in 2000. Proposed site-testing was based on two kinds of measurements. First, a monitoring of the turbulence parameters in the visible (seeing $r_{0}$, isoplanatic angle $\theta_{0}$, outer scale $\mathcal{L}_{0}$, and coherence time $\tau_{0}$ ) with a GSM experiment (Ziad et al. 2000) specially designed to work in polar winter conditions. In addition to this monitoring, it was proposed to launch balloons equipped with microthermal sensors to measure the vertical profile of the refractive index structure constant $C_{n}^{2}(h)$ (Barletti et al. 1977). Is was predicted that 50 to 60 balloons would be regularly launched during the polar winter.

On-site campaigns began in summer 2000-2001 and were performed every year until 2004-2005 with a double aim. It was first necessary to test the behaviour of all instruments in the "intermediate" cold temperature of the summer season that ranges between -20 and $-50{ }^{\circ} \mathrm{C}$. This first step was also used to anticipate the more difficult winter conditions, with -50 to $-80{ }^{\circ} \mathrm{C}$, in order to be technically confident of the first winterover equipment. On the other hand, the summertime sky quality is interesting in itself, so solar observations were started in 1979-1980 at the South Pole. At Dome C the long uninterrupted sequences of coronal sky (far longer than at South Pole) and the expected occurrence of excellent seeing make it a very promising site for high resolution solar imaging and specially solar coronography.

So far, 6 summer campaigns have been performed totalling 80 man-weeks of presence on the site. The first winterover also began this year. 197 meteorological balloons were successfully launched and corresponding results were published in Aristidi et al. (2005). Major conclusions are that the wind speed profiles in Dome $\mathrm{C}$ appear as the most stable among all the astronomical sites ever tested, and that the major part of the atmospheric turbulence is probably generated in the first $100 \mathrm{~m}$ above the snow surface, where the temperature gradients are the steepest (around $0.1{ }^{\circ} \mathrm{C} / \mathrm{m}$ ).

In this paper we present the results of daytime turbulence measurements (seeing and isoplanatic angle) made with various techniques. The paper is organised as follows: in Sect. 2 we 
briefly review the theory of the turbulence parameters we measured. Section 3 presents the instrumental setup at Dome C. Section 4 describes the observations, the various calibration procedures, and the online and offline data processing. The results of the monitoring are in Sect. 5. A final discussion is presented in Sect. 6 and an appendix on error analysis ends the paper.

\section{Theory}

\subsection{Seeing}

Atmospheric turbulence is responsible for the degradation of image resolution when observing astronomical objects. The full width at half maximum (FWHM) of the long-exposure point-spread function broadens to a value $\epsilon$ called seeing, usually expressed in arcseconds; this parameter represents the angular resolution of images for given atmospheric conditions. In the visible, $\epsilon$ is around $1^{\prime \prime}$ for standard sites.

Fried (1966) introduced a so-called parameter $r_{0}$ that can be regarded as the diameter of a telescope whose Airy disc has the same size as the seeing. He derived the following relation

$\epsilon=0.98 \frac{\lambda}{r_{0}}$

The seeing is one of the most important parameters that describe atmospherical turbulence. Seeing monitors have been installed in major observatories, such as ESO Paranal, and they produce constant data that are used to optimize the observations. Seeing estimation can be made by various methods (Vernin \& Munoz 1995); seeing monitors that allows continuous measurements are traditionally based on differential image motion, such as the DIMM (Differential Image Motion Monitor) used at Dome C. It is extensively described in the literature (Sarazin \& Roddier 1990; Vernin \& Munoz 1995; Tokovinin 2002) and has become very popular because of its simplicity.

A DIMM is a telescope with an entrance pupil made of 2 diffraction-limited circular sub-apertures of diameter $D<r_{0}$, separated by a distance $B>D$. A tilt is given to the light propagating through one of the two apertures to produce twin images that move according to the turbulence. The Fried parameter is computed from longitudinal $\left(\sigma_{l}^{2}\right)$ and transversal $\left(\sigma_{t}^{2}\right)$ variances of the image motions using Eqs. (5) and (8) of Tokovinin (2002).

\subsection{Isoplanatic angle}

The isoplanatic angle is a fundamental parameter for adaptive optics (AO). It is the correlation angle of the turbulence, i.e. the maximum angular distance between two point-sources affected by the same wavefront distortions. In AO systems, these distortions are usually estimated on a nearby bright reference star. This reference star must be in the isoplanatic domain, which in most cases dramatically reduces the observable piece of sky.

As for the seeing, the isoplanatic angle $\theta_{0}$ is a scalar random variable, usually expressed in arcseconds, resulting from an integral over the $C_{n}^{2}$ profile (Ziad et al. 2000;

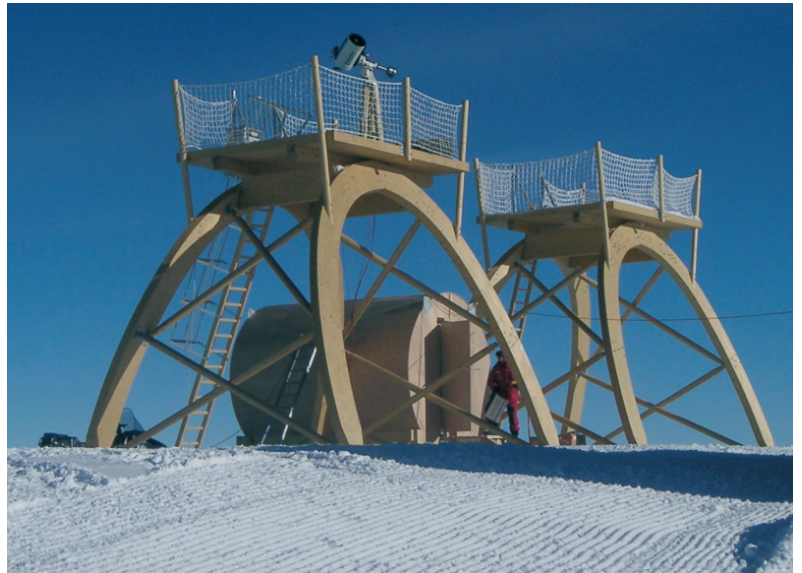

Fig. 1. Concordiastro observatory in November 2004. One can see the two platforms and the wooden igloo between. The DIMM is on the top of one platform.

Avila et al. 1998). Loos \& Hogge (1979) proposed an approximate estimation based on the scintillation of a point-source star through a $10-\mathrm{cm}$ pupil. Estimation is even better if one uses a 4-cm diameter central obstruction. As for the DIMM, this technique allows continuous monitoring of the isoplanatic angle, as well as of the scintillation data. It is used routinely by the GSM instrument (Ziad et al. 2000) for site qualification.

\section{Instrumentation}

\subsection{The Concordiastro observatory}

The Concordiastro observatory is based on two wooden platforms designed by J. Dubourg (Observatoire de la Côte d'Azur) and built by the "Ateliers Perrault Frères", a factory in western France. The design of these platforms recalls the first floor of the Eiffel Tower (see Fig. 1). These platforms are $5 \mathrm{~m}$ high and equipped with massive supports for the telescopes. The height has been chosen for site-testing purposes to avoid the surface-layer turbulence. They are located $300 \mathrm{~m}$ away from the Concordia station, in a SW direction to avoid pollution from the prevailing S or SW winds (Aristidi et al. 2005). The first one was erected in December 2002, the second in January 2004. All the installation is built onto a $2 \mathrm{~m}$ high pavement of compacted snow for stability, as for the Concordia buildings.

Between the two platforms, a wooden container nicknamed "igloo" hosts the electronics and the control systems. It was installed at the very end of the 2003-2004 summer campaign, and cabled to the telescopes. A fiber optic link is foreseen for remote control from Concordia towers during the winter.

Additional telescopes have been installed at $1.50 \mathrm{~m}$ above the ground during the campaign 2004-2005 for estimating the surface layer contribution in the seeing.

\subsection{Telescopes}

We use Schmidt-Cassegrain Celestron C11 telescopes (diameter $280 \mathrm{~mm}$ ) with a $\times 2$ Barlow lens (equivalent focal length $5600 \mathrm{~mm}$ ). Optical tubes have been rebuilt in INVAR to reduce 
Table 1. Technical specifications of the CCD camera.

\begin{tabular}{ll}
\hline \hline Number of pixels: & $640 \times 480$ \\
Pixel size: & $9.9 \times 9.9 \mu \mathrm{m}$ \\
Binning modes: & horizontal: 1,2 \\
& vertical: $1,2,4$ \\
Dynamic range: & 12 bits \\
Exposure time: & $10 \mu$ s to $10 \mathrm{~s}$ \\
Frame rate & $40 \mathrm{fps}$ without binning \\
& $76 \mathrm{fps}$ in binning $2 \times 2$ \\
Maximum QE & $40 \%$ at 350 and $500 \mathrm{~nm}$ \\
Bandwith (FWHM) & $320-630 \mathrm{~nm}$ \\
ADU & $7 \mathrm{e}^{-} /$count \\
Readout noise: & $16 \mathrm{e}^{-}$ \\
\hline
\end{tabular}

thermal dilatations, which cause defocus since they change the distance between the primary and secondary mirrors. Several technical improvements were made on the primary mirror support, and the grease of the focus system has been replaced by a cold-resistant one (up to $-90^{\circ} \mathrm{C}$ ).

These telescopes were placed on equatorial mounts AstroPhysics 900. As for the telescope, the mounting also needed to be customised to the climate: its grease had to be changed and heating systems placed in the motor carters. Tracking worked successfully during the entire polar summers. The mounts are placed on massive wooden feet fixed to the platform. Polar alignment is made by Bigourdan's method on solar spots (fortunately we were close to a solar maximum so finding spots had never been a problem), then on Venus, and fine tuning was made on Canopus itself during the observations.

\subsection{Cameras}

At the focus of all our telescopes we use a digital CCD camera (PCO Pixelfly) connected to a PCI board via a high speed transfer cable. Technical specifications are given in Table 1. The camera was placed into an insulated and thermally controlled box, with the insulation inherited from spatial technology. Typical temperature inside the box was around $-15{ }^{\circ} \mathrm{C}$, and over $0{ }^{\circ} \mathrm{C}$ on the $\mathrm{CCD}$ chip thanks to dissipation. The $\times 2$ Barlow lens was placed at the box entrance.

\section{Observations and data processing}

\subsection{Seeing measurements with the DIMM}

Dome C DIMM is based on a Celestron 11 telescope equipped with a 2-hole mask at its entrance pupil. Each hole has a diameter of $6 \mathrm{~cm}$ and is separated by $20 \mathrm{~cm}$ from the other one. One is equipped with a glass prism giving a small deviation angle ( 1 arcmin) to the incident light. The other is simply closed with a glass parallel plate. The size of the Airy disc is $\lambda / D=40 \mu \mathrm{m}$ at the operating wavelength (visible), which is compatible with Shannon sampling in $2 \times 2$ binning mode, with effective pixel size of $20 \mu \mathrm{m}$. The separation of the two images in the focal plane is $1.6 \mathrm{~mm}$ ( 80 pixels).

After different trials, we selected the star Canopus ( $\alpha$ Car, $V=-0.7$ ) for seeing monitoring. It is circumpolar at Dome $\mathrm{C}$

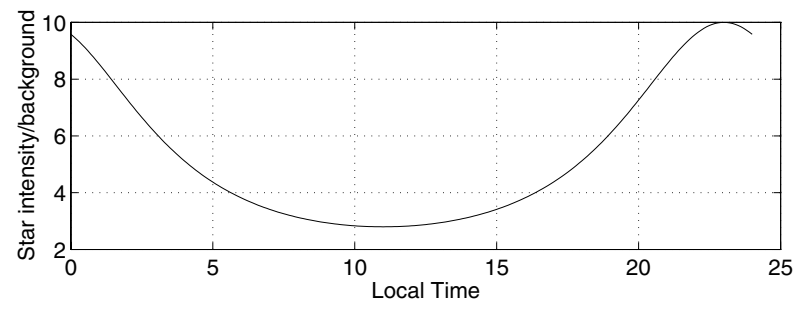

Fig. 2. Plot of the star image peak intensity $I_{m}$ to the sky background level model $\langle B(t)\rangle$. From images taken in the period Dec. 1-15, 2003.

with zenithal angles $z$ ranging between $22^{\circ}$ and $52^{\circ}$. At the end of December, Canopus and the Sun have a 12-h difference in right ascension, so that Canopus is at its maximum (resp. minimum) elevation when the Sun is at its minimum (resp. maximum). The angular distance between the two bodies remains around $100^{\circ}$ during the whole summer season.

Three DIMM campaigns have been performed so far. The first one in 2002-2003 (Aristidi et al. 2003a,b) led to seeing values that since have seemed over-estimated. The telescope used was black so heated by the Sun: we had strong local turbulence that sometimes destroyed the Airy discs of the star images into speckle patterns. We noticed evidence of this local turbulence by a posteriori comparison between data taken simultaneously from white and black telescopes. The 2002-2003 data will therefore not be taken into account in this paper.

The seeing values presented here were collected during the periods of Nov. 21, 2003 to Feb. 2, 2004 and Dec. 4, 2004 to Feb. 28, 2005. Data are also available beyond March 2005, but this paper deals with summer conditions, so we limited the data sample to the daytime. Autumn and winter seeing will be discussed in forthcoming papers.

\subsubsection{Sky background}

The sky background level is a strong limitation in daytime stellar observations. We decided to quantify this background in the first half of December 2003. From each image taken in that period, we measured the sky background $B(t)$ as a function of local time $t$. We then performed a sinusoidal fit giving an empirical model for the mean sky background $\langle B(t)\rangle$ as a function of local time. We also measured and averaged the peak intensity of the star images $I_{m}$. Figure 2 plots the ratio $I_{m} /\langle B(t)\rangle$ as a function of the local time. Background level is always between $10 \%$ and $30 \%$ of the stellar flux, which is low enough to apply a threshold and still keep enough stellar flux to make measurements. We were then able to perform the first, to our knowledge, DIMM measurements ever in daytime, which can be credited to the exceptional quality of the Dome C sky appearing coronal a large fraction of the time.

\subsubsection{Exposure time}

The Fried's parameter must be ideally expressed for instantaneous images. As a finite exposure time is used by the camera, there is an exposure bias that must be removed. The technique, 
described by Tokovinin (2002), consists in making successive poses using alternate exposure times $\tau, \tau / 2, \tau, \tau / 2, \ldots$ and performing a modified exponential extrapolation to attain instantaneous values:

$\epsilon(0)=\epsilon(\tau)^{1.75} \epsilon(\tau / 2)^{-0.75}$

where $\epsilon(x)$ is the seeing estimated with exposure time $x$. We chose $\tau=10 \mathrm{~ms}$, which had the double advantage of exploiting the entire CCD dynamics and being a standard for seeing monitors. We observed that the correction depends on the turbulence conditions; it is close to zero when the seeing is good and can grow up to $20 \%$ when $\epsilon>1.5^{\prime \prime}$. A few percent difference between transverse and longitudinal seeing values has also been noticed; it is a well-known effect (Martin 1987) related to the wind speed and direction and to the exposure time.

\subsubsection{Seeing estimations}

Times series were divided into 2-min intervals in which around 9000 short-exposure frames were acquired using the $2 \times 2$ binning mode of the CCD. $2 \mathrm{~min}$ is a long enough time interval to saturate the structure function of the motion of DIMM images. Software was developed to perform real time data processing. Each short exposure frame was flat-fielded to eliminate the background, then the two stellar images were easily detected in two small $20 \times 20$ pixel windows, and their photocentre coordinates computed by means of a simple barycenter formula. Note that with the flat-fielding, the effective illuminated pixels correspond roughly to the surface of the Airy disc of the sub-apertures. Every two minutes, the variances of longitudinal and transverse distances between the two images were computed in units of pixel square, then converted into arcsec using the scale calibration described below. This leads to two independent estimates of the Fried parameter, namely $r_{0 l}$ (longitudinal) and $r_{0 t}$ (transverse), which are stored in a file. Then the two $20 \times 20$ pixel windows are moved so that their centre is placed on the previous photocentres of the two star's images for the following seeing estimation. This allows a gain of time in the barycentre calculation, and allows the stars to be followed if they move in the field of view (guiding problems for example).

Three corrections are then made in post-processing to obtain actual seeing values:

- Transversal and longitudinal seeings are computed and corrected from exposure time, as described above.

- As the seeing is a scalar parameter, both transverse and longitudinal estimations should give the same value. We kept only pairs verifying $0.7<\epsilon_{t} / \epsilon_{l}<1.3$ (around $90 \%$ of the data sample). Longitudinal and transverse values are then averaged.

- Finally we compensated from zenithal distance $z$ (Tokovinin 2002).

\subsubsection{Scale calibration}

The differential variances are obtained in units of pixel square and require calibration of the pixel size. This was done by making image sequences of the star $\alpha$ Centauri. It is an orbital bright binary star, whose angular separation (around 10") was computed from its last orbit (Pourbaix et al. 2002).

Average autocorrelation of the images of the binary were computed to reduce noise (one image sequence is around 600 images). This kind of processing is well known in speckle interferometry to measure double-star separation. This function exhibits 3 peaks whose distance is the separation of the binary stars in pixels. This gave a pixel scale of $\xi=0.684 \pm 0.004^{\prime \prime}$ (with binning $2 \times 2$ ).

\subsubsection{Strehl ratio of DIMM images}

The Strehl ratio is an estimator of the quality of the two stellar images produced by the DIMM. It is the ratio of the star's image intensity at its maximum to the intensity of the theoretical Airy disc that would have been obtained in perfect conditions. The Strehl ratio is affected by fixed aberrations, as well as optical turbulence. It is generally assumed that image quality is good when the Strehl ratio is over $30 \%$.

Monitoring the Strehl ratio of the two stellar images produced by a DIMM can provide an image selection criterion. A simple calculation formula has been proposed by Tokovinin (2002). Though continuous monitoring of the Strehl is not implemented in the data acquisition software, we performed an a posteriori estimation of the Strehl ratio of our DIMM images in typical conditions. From data taken in the 6-day period of 10-15 December 2004, we estimated the Strehl ratio of the two stellar images for each short-exposure frame. We collected around 3400000 values and found average Strehl ratios $\left\langle S_{l}\right\rangle=$ $0.56 \pm 0.11$ for the image on the left and $\left\langle S_{r}\right\rangle=0.53 \pm 0.11$ for the one on the right. These values indicate good image quality. Airy rings around the twin images were indeed often observed with the DIMM's eyepiece.

\subsection{Isoplanatic angle measurements}

The isoplanatic angle was monitored during the month of January 2004. As for the DIMM, the telescope used for monitoring the isoplanatic angle is a Celestron C11. A mask with a $10 \mathrm{~cm}$ aperture and $4 \mathrm{~cm}$ central obstruction was placed at the entrance pupil. Monitoring was performed from Jan. 5 to Feb. 2, 2004.

The observing procedure was similar to the DIMM. The same star, Canopus, was used along with exposure times from $\tau=8$ to $12 \mathrm{~ms}$. To compensate for exposure bias, we alternated frames with exposure times $\tau$ and $\tau / 2$. Time series were divided into 2-mn intervals. For each short-exposure frame the following operations were applied:

- Background mean level $\bar{b}$ was estimated on the whole image, then subtracted.

- Low level values were set to zero. A threshold was chosen at $5 \sigma_{b}$, with $\sigma_{b}$ the background variance.

- After these operations the star image was spread over $N_{I} \simeq$ 100 pixels in $2 \times 2$ binning mode and $N_{I} \simeq 250$ pixels without binning. Total stellar flux $I$ was estimated by integration over these illuminated pixels.

- Values of $I, \bar{b}, \sigma_{b}$, and $N_{I}$ were logged in a file. 


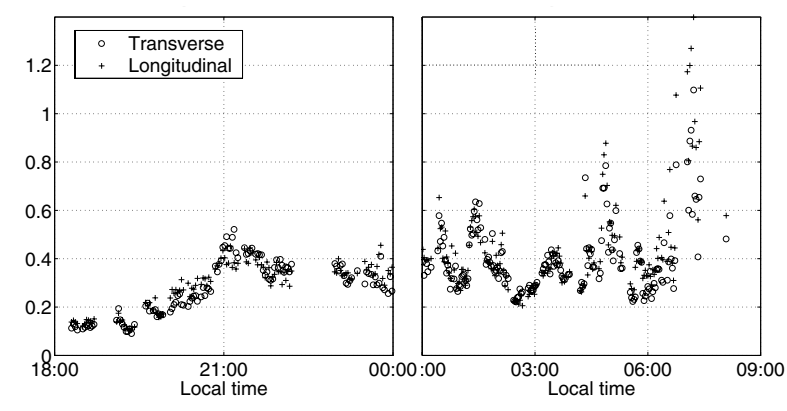

Fig. 3. First seeing curves obtained during the 2003-2004 campaign on November 21 and 22, 2003. We show here the longitudinal (+) and transverse (o) time series.

A 2-min sequence corresponds to $N \simeq 3300$ images in $2 \times 2$ binning mode, and to $N \simeq 1400$ images without binning. One sequence leads to one value of the isoplanatic angle, computed as post-processing following the algorithm described as:

- Separation of the values corresponding to exposure times $\tau$ and $\tau / 2$ in two subsets.

- On each subset, computation of $\bar{I}, \sigma_{I}$, and scintillation indexes $s_{\tau}$ and $s_{\tau / 2}$.

- Compensation for exposure time by linear extrapolation (Ziad et al. 2000).

- Calculation of $\theta_{0}$ for $\lambda=0.5 \mu \mathrm{m}$.

\section{Results}

\subsection{Seeing monitoring}

A total amount of 31597 2-min seeing values were been estimated during the 2003-2004 and 2004-2005 campaigns. Thanks to the presence of two observers, several long-time series were made possible, making the monitoring as continuous as possible. As the polar alignment was progressively improved, we could then leave the telescope alone almost $8 \mathrm{~h}$ without losing the star. Figure 4 (top) shows the number of seeing values obtained every day during the two campaigns ( $m a x-$ imum possible value is 720). Several periods of no measurements were due to bad weather (as in December 2003, where we had 10 successive days of covered sky) or logistics. This was the case in mid-January 2005 where part of the equipment was transferred into the Concordia buildings, and some work was done to set up the remote control.

Amazingly low seeing values were observed during the first days of the 2003-04 campaign. November 21 corresponds to spring in the Southern hemisphere; the temperature was then close to $-50{ }^{\circ} \mathrm{C}$. These temperature conditions are the closest to winter values we had on the 3 campaigns. The first 2-day seeing time series are shown in Fig. 3. Exceptional seeing as low as $0.1^{\prime \prime}$ was observed, and we had a continuous period of $10 \mathrm{~h}$ of seeing below $0.6^{\prime \prime}$. Daily median values are plotted in Fig. 4.

Seeing statistics are summarised in Table 2. As mentioned above, all measurements are computed at $\lambda=500 \mathrm{~nm}$ in daytime. Seeing values are in arcsec. Seeing histograms are displayed in Fig. 5. Scale was set to logarithmic (base 10) on the
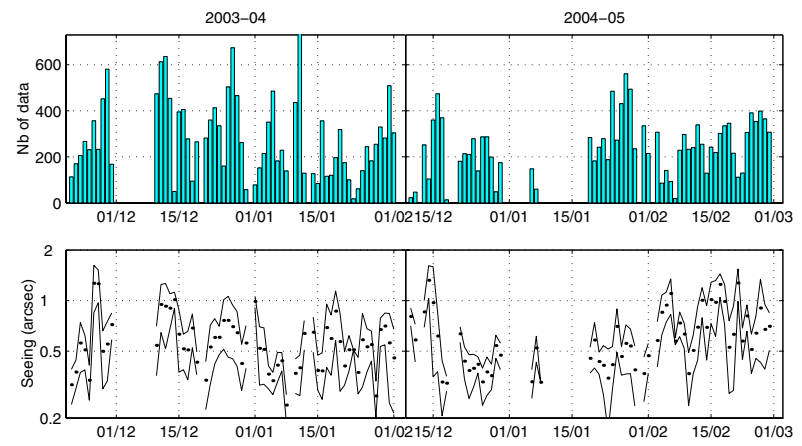

Fig. 4. Top: number of seeing data per day. Bottom: points are daily median seeing values and intervals containing $50 \%$ of values is delimited by lines. Data collected during the last two summer campaigns. Seeing axis is logarithmic

Table 2. Seeing statistics for the two summer campaigns. These numbers stand for the DIMM at $h=8.5 \mathrm{~m}$.

\begin{tabular}{lrrr}
\hline \hline Campaign & $2003-04$ & $2004-05$ & Total \\
\hline Number of measurements & 17128 & 14469 & 31597 \\
Median seeing (") & 0.54 & 0.55 & 0.55 \\
Mean seeing (") & 0.65 & 0.67 & 0.66 \\
Standard deviation (") & 0.39 & 0.38 & 0.39 \\
Max seeing (") & 5.22 & 3.33 & 5.22 \\
Min seeing (") & 0.10 & 0.08 & 0.08 \\
\hline
\end{tabular}

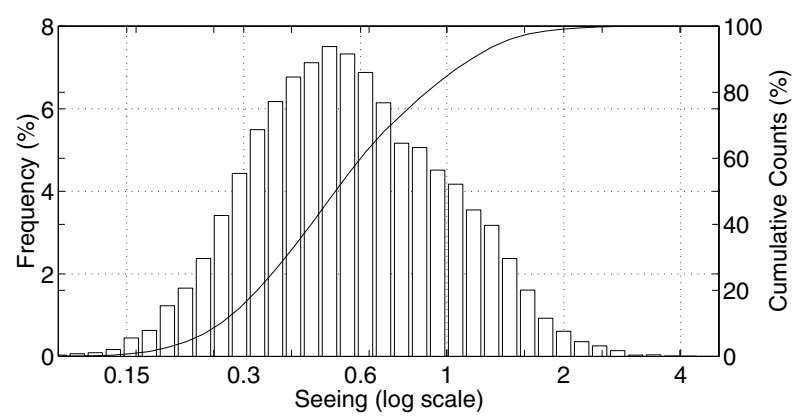

Fig. 5. Histogram (stairs) and cumulative histogram (continuous line) of seeing values for the 2003-2004 and 2004-2005 campaigns. Seeing axis is in logarithmic scale.

seeing axis to emphasize the log-normal distribution of the values. The $50 \%$ percentile (the median) is at $\epsilon=0.55^{\prime \prime}$ : seeing is then better than $0.55^{\prime \prime}$ half of the time.

These values are exceptionally good for daytime seeing, when the Sun is present in the sky and heats the surface. It can compare with night-time seeing of the best observatories. Table 3 shows a comparison with other sites.

Another interesting result is the behaviour of the seeing with time. Figure 6 has been calculated by binning all seeing values into 30 -min intervals. Best seeing values, like $0.4^{\prime \prime}$ or better, are generally obtained in mid local afternoon. It is extremely encouraging for solar imaging at high angular resolution. Indeed, a discontinuity of the temperature gradient between 200 and $400 \mathrm{~m}$ has often been noted in the middle of the day, and disappears in the evening to be replaced by a standard surface inversion layer of 20 or $30 \mathrm{~m}$ (Aristidi et al. 2005). 
Table 3. Comparison of Dome C daytime seeing with daytime and night-time seeing in other observatories.

\begin{tabular}{llllll}
\hline \hline Daytime values & & & Night-time values & & \\
\hline Site & Seeing & Reference & Site & Seeing & Reference \\
\hline White Sands & 2.24 & Walters et al. (1979) & Paranal & 0.66 & Sarazin (www.eso.org/ msarazin) \\
Sac Peak & 1.68 & Ricort et al. (1979) & Mauna Kea & 0.63 & Tokovinin et al. (2005) \\
Roque de Muchachos & 1.91 & Borgnino \& Brandt (1982) & Roque de Muchachos & 0.64 & Muñoz et al. (1997) \\
Sac Peak & 1.16 & Brandt et al (1987) & Cerro Pachon & 0.89 & Ziad et al. (2000) \\
Calern & 2.5 & Irbah et al. (1994) & Maidanak & 0.69 & Eghamberdiev et al. (2000) \\
Fuxian Lake & 1.20 & Beckers \& Zhong (1994) & South Pole & 1.74 & Travouillon et al. (2003) \\
\hline Dome C & 0.54 & this paper & Dome C (day) & 0.54 & this paper \\
\hline
\end{tabular}
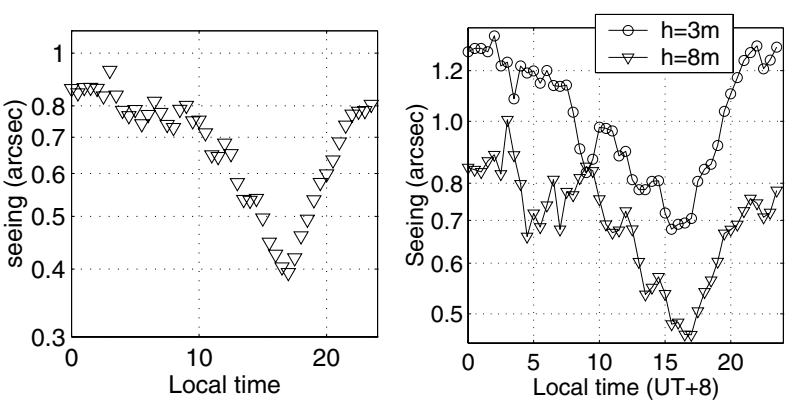

Fig. 6. Left: seeing versus time, averaged over the 2003-04 and 2004-05 campaigns. Seeing values, obtained from the DIMM at elevation $h=8 \mathrm{~m}$, were binned into 30-min intervals. Right: seeing versus time in 2004-2005 for the two DIMMs.

During the afternoon transition, there is a moment with an isothermal temperature profile. The generally excellent seeing obtained during this transition indicates that the contribution of all the rest of the atmosphere is indeed very small. At night with a telescope standing above the ground inversion layer, a really excellent seeing could then be expected almost continuously. The height of this inversion layer is an open question to be answered after the winterover.

\subsubsection{Contribution of the surface layer}

During the 2004-05 summer campaign, the presence of two DIMMs observing simultaneously at two different heights $(3 \mathrm{~m}$ and $8 \mathrm{~m}$ over the plateau snow surface) allowed investigation of the contribution of the surface layer to the seeing. Radiosoundings had already shown that the strongest thermal gradients are observed close to the surface (Aristidi et al. 2005), and an important part of the turbulence is expected to be generated in the first tens of meters.

All the telescopes were installed in the first days of December 2004, and the seeing monitoring started for telescope 1 (on the ground) and 3 (on the platform) on December 10. Median/mean seeings are $0.55 / 0.67$ " for telescope at $8 \mathrm{~m}$ and $0.93 / 1.03^{\prime \prime}$ for telescope at $3 \mathrm{~m}$ (statistics over 15000 values obtained in December 2004, January and February 2005). There is an big difference which appears to be time-dependent. Figure 6 shows the behaviour of the seeing measured at the two heights as a function of local time. Both curves exhibit a noticeable minimum in mid-afternoon, though less pronounced for the $3 \mathrm{~m}$ curve.
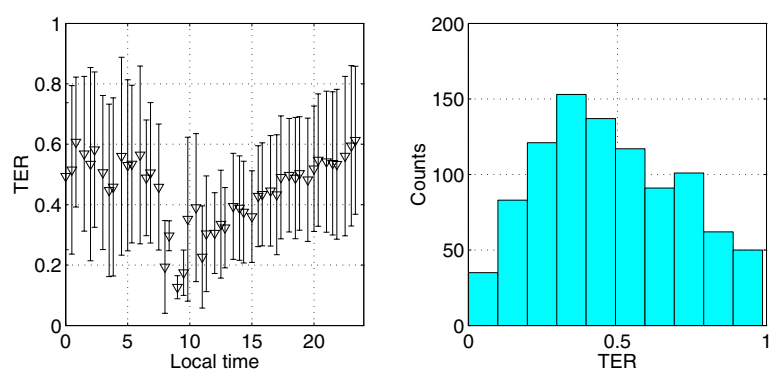

Fig. 7. Left: surface layer turbulent energy ratio $(T E R)$ as a function of time. Error bars are the standard deviation of the sample distribution. Right: TER histogram.

We can describe the surface layer contribution with a turbulent energy ratio (TER), following Martin et al. (2000):

$T E R=\frac{\int_{3 \mathrm{~m}}^{8 \mathrm{~m}} C_{n}^{2}(h) \mathrm{d} h}{\int_{3 \mathrm{~m}}^{\infty} C_{n}^{2}(h) \mathrm{d} h}$.

This TER gives the ratio of the turbulent energy in the $5 \mathrm{~m}$ surface layer to the total turbulent energy (integrated from $3 \mathrm{~m}$ to infinity). These integral can be estimated from the seeing through the Fried's parameter (Roddier 1981). The TER is then given by

$$
T E R=\frac{r_{0}(3 m)^{-5 / 3}-r_{0}(8 m)^{-5 / 3}}{r_{0}(3 m)^{-5 / 3}} .
$$

The TER was calculated every time the two telescopes were operated simultaneously. Its histogram and time-dependence are shown in Fig. 7. The mean value of $48 \%$ indicates that almost half of the ground turbulence is concentrated into the first $5 \mathrm{~m}$ above the surface.

\subsection{Isoplanatic angle and scintillation}

More than 6000 values of the isoplanatic angle were collected during the month of January, 2004. Statistics of both $\theta_{0}$ and the scintillation index $s$ summarised in Table 4 show a median value $\theta_{0}=6.8^{\prime \prime}$ at wavelength $\lambda=0.5 \mu \mathrm{m}$, which is far better than values obtained at any astronomical site (see Table 5 for a comparison). Good values are also found at the South Pole where the atmosphere above the first $220 \mathrm{~m}$ is calm (Marks et al. 1999); the isoplanatic angle is indeed more sensitive to high altitude turbulence. Winter estimates given by the MASS in the AASTINO station (Lawrence et al. 2004) indicates values similar to ours, despite the presence in winter of 
Table 4. Isoplanatic angle $\left(\theta_{0}\right)$ and scintillation index statistics during the month of January 2004.

\begin{tabular}{lrr}
\hline \hline & $\theta_{0}\left({ }^{\prime \prime}\right)$ & $s(\%)$ \\
\hline \# of measurements & 6368 & 6368 \\
Mean value & 6.8 & 0.88 \\
Median value & 6.8 & 0.63 \\
standard deviation & 2.4 & 0.90 \\
Min value & 0.7 & \\
Max value & 17.1 & \\
\hline
\end{tabular}

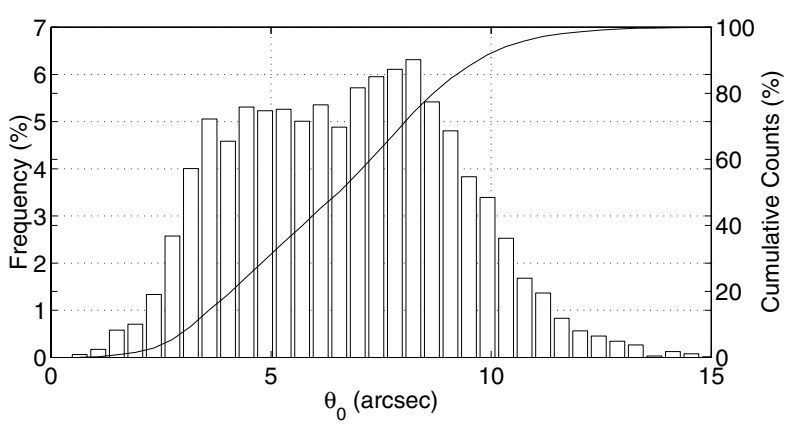

Fig. 8. Histogram (stairs) and cumulative histogram (line) of isoplanatic angle values for the campaign 2003-2004.
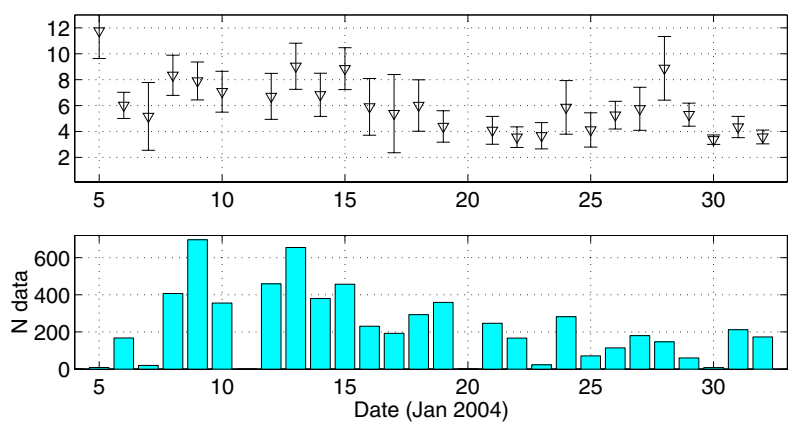

Fig. 9. Isoplanatic angle as a function of day. Top: daily median values. Error bars correspond to the $1 \sigma$ dispersion of the daily values. Bottom: number of data per day.

high-altitude winds on the order of $40 \mathrm{~m} / \mathrm{s}$ (Aristidi et al. 2005 and references therein).

The histogram and cumulative histogram are shown in Fig. 8, time series in Fig. 9. The daily median values plot at the top of Fig. 9 shows a small degradation of $\bar{\theta}_{0}$ between the beginning and the end of January.

The high value of isoplanatic angle, roughly 3 times higher than at classical sites, is good news for adaptive optics. This corresponds to a gain of a factor 10 in the field used to find calibrator stars, which therefore increases the observable piece of sky, as discussed by Coudé du Foresto et al. (2004). Another advantage of a large isoplanatic domain is the uselessness of multi-conjugate adaptive optics for high-resolution wide field imaging (Lawrence 2004).

\section{Discussion and conclusion}

We have presented the results of optical turbulence measurements during two summer campaigns at Dome C. The main
Table 5. Comparison of Dome $\mathrm{C}$ isoplanatic angle with values observed at other sites.

\begin{tabular}{lll}
\hline \hline Site & $\theta_{0}$ & Reference \\
\hline Paranal & 1.91 & Ziad et al. (2000) \\
La Silla & 1.25 & Ziad et al. (2000) \\
Cerro Pachon (Chile) & 2.71 & Ziad et al. (2000) \\
Maidanak & 2.47 & Ziad et al. (2000) \\
Oukaimeden (Morocco) & 1.58 & Ziad et al. (2000) \\
South Pole & 3.23 & Marks et al. (1999) \\
Dome C & 6.8 & this paper \\
\hline
\end{tabular}

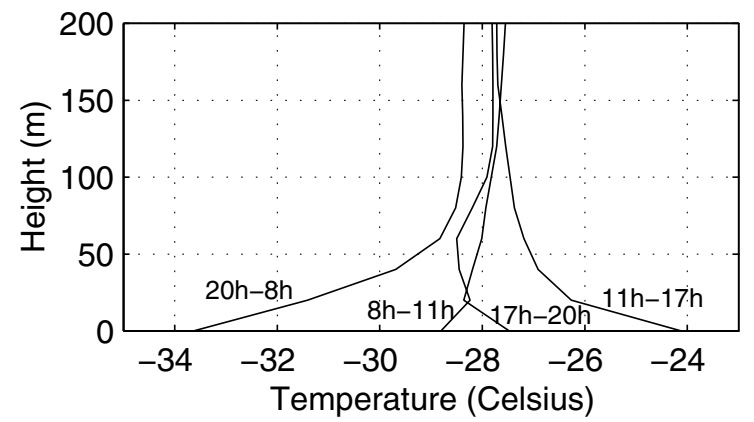

Fig. 10. Mean temperature profiles above the ground (from Aristidi et al. 2005), based on in-situ radiosoundings. On the vertical axis, height is counted from the snow (altitude $3260 \mathrm{~m}$ ). The four curves correspond to measurements performed at four different times of the day.

result is the exceptional seeing quality in the daytime, allowing image resolution better than $0.5^{\prime \prime}$ during a few hours every day, and the large value of the isoplanatic angle, three times larger than Mt Paranal in night-time. Combining this with long periods of clear and coronal sky makes Dome $\mathrm{C}$ probably one of the best sites on earth for solar visible and infrared astronomy.

We recently published (Aristidi et al. 2005) a study based on balloon-borne meteo radio-sondes launched during 4 summer seasons, allowing us to make statistics on wind speed and temperature profiles in the atmosphere above Dome C. Among the numerous results presented in that paper, we found that the temperature profile exhibits a strong gradient in the boundary layer (the first $100 \mathrm{~m}$ above the snow). The corresponding curve is shown in Fig. 10. This gradient, positive at midnight (ice is cooler than the air above) and negative at noon (ice is heated by the Sun radiation), vanishes twice a day: in the morning and near $5 \mathrm{pm}$. Seeing indeed appears to be the best during the afternoon near $5 \mathrm{pm}$. The other expected seeing minimum in the morning was sometimes observed, especially in the 2003-2004 campaign. But it does not appear in the daily averaged curves displayed in Fig. 6, and at this time we have no convincing explanation for that.

This behaviour of the boundary layer temperature profile and of the seeing-versus-time curve suggests that the turbulence is dominated by the first tens of meters above the ground. This is the same at the South Pole, where the height of this very turbulent boundary layer is $220 \mathrm{~m}$ (Marks et al. 1999). Indeed, another indicator is the correlation between the seeing and the isoplanatic angle. Both result from an integral over the whole 


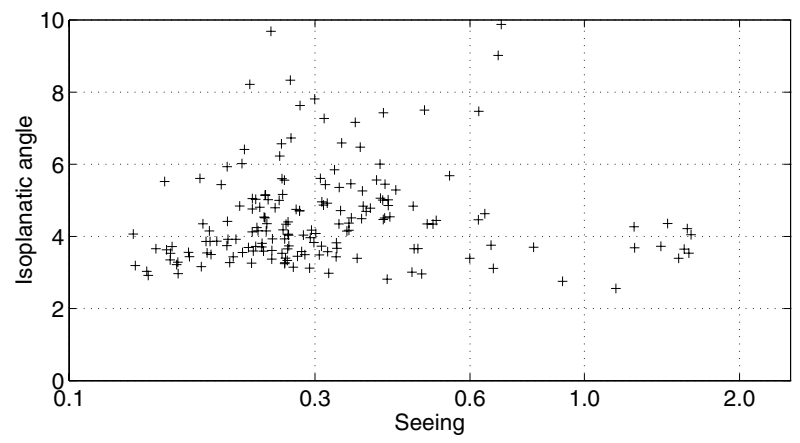

Fig. 11. Plot of the isoplanatic angle versus seeing (data collected in January 2004).

atmosphere, but the isoplanatic angle is more sensitive to high turbulent layers (ponderation by $h^{5 / 3}$ in the integral definition of $\theta_{0}$ (Ziad et al. 2000)). Figure 11 displays a plot of the isoplanatic angle versus seeing showing no dependence between the two parameters. Quantitative estimations of the surface layer contribution have been made possible in 2004-2005 with the presence of two DIMMs. $50 \%$ of the ground seeing is generated in the first $5 \mathrm{~m}$.

The winter measurements are now being awaited with a lot of excitement, to know how much residual turbulence will exist above this ground layer during the winter night, how thick this layer will be, and how much turbulence will exist below. The last summer campaign was the first one to be immediately followed by the historical first winterover, and Agabi has volunteered to spend one year at Concordia to conduct the observations. Seeing and isoplanatic angle monitoring are in progress. In-situ soundings of the vertical profile of $C_{n}^{2}$ by means of balloon-borne microthermal sensors are also at the menu for the winter. They will give access to parameters such as the outer scale and coherence time. Finally, a monitoring of $C_{n}^{2}$ in the boundary layer is also foreseen, using a ground version of the balloon experiment which takes advantage of the $32 \mathrm{~m}$ high American tower.

At the time of writing this paper, the night is getting longer and longer every day, and these questions will receive firm answers quite soon.

Acknowledgements. We wish to acknowledge both Polar Institutes, IPEV and ENEA, for funding the programme and for the logistics support. Our thanks go to all the Concordia staff at Dome $\mathrm{C}$ for their friendly and efficient help in setting up the Concordiastro platforms. We are also grateful to our industrial partners "Optique et Vision" and "Astro-Physics" for technical improvement on the telescopes and their mounts to make them work in polar conditions. Finally we thank JeanMichel Clausse and Jean-Louis Dubourg who were present on site during the campaings 2000-2001 and 2001-2002.

\section{Appendix A: Error analysis}

\section{A.1. Seeing}

Statistical error. Variance of image motion is computed from a sample of $N \simeq 9000$ individual frames: it is then affected by statistical noise due to the finite size of the sample. Assuming statistical independence between the frames, the statistical error on the variance $\sigma^{2}$ is given by (Frieden 1983; Sarazin \& Roddier 1990)

$\frac{\delta_{\mathrm{s}} \sigma^{2}}{\sigma^{2}}=\sqrt{\frac{2}{N-1}}$

that propagates an error contribution $\delta_{\epsilon}$ onto the seeing. With 9000 independent frames, we have $\frac{\delta_{\mathrm{s}} \sigma^{2}}{\sigma^{2}}=1.4 \%$ and $\frac{\delta_{\mathrm{s}} \epsilon}{\epsilon}=$ $0.9 \%$. Frames are not independent at our sampling rate, and this is only a lower boundary.

Scale error. Image motion is converted from pixels to arcsec using the factor $\xi$ introduced before. The uncertainty on $\xi$ propagates into the differential variances when the conversion from pixels into arcsec is performed. With actual value $\frac{\delta \xi}{\xi}=0.006$, this gives a relative contribution on the differential variances $\frac{\delta_{\mathrm{p}} \sigma^{2}}{\sigma^{2}}=1.2 \%$ and on the seeing $\frac{\delta_{\mathrm{p}} \epsilon}{\epsilon}=0.7 \%$.

Readout noise Influence of the CCD readout noise on DIMM data is discussed in Tokovinin (2002). The readout noise is a random independent contribution to the measured flux. It biases the computed differential variances by a term

$\sigma_{R}^{2}=2 \frac{R^{2}}{I^{2}} \sum_{\text {window }} x_{i j}^{2}$

where $I$ is the total stellar flux, $R$ the readout noise (2.3 ADU for the Pixelfly) and $x_{i j}$ the coordinates of contributing pixels. The number of illuminated pixels is on the order of 30 after flat fielding, which defines the "window" over which the summation is made. The order of magnitude of this bias in our case is $\sigma_{R}^{2} \simeq 10^{-6}$ square pixels. Comparing this value to our standard differential variances ( 0.1 to 1 square pixel), we can see that the readout noise bias is extremely small and can be neglected.

Background noise. The sky background is an additive Poisson noise independent of the stellar signal. Therefore its effects on the differential centroid variance is the same as the readout noise: a bias term $\sigma_{B}^{2}$. It can be computed using Eq. (A.2), substituting $R$ by $B$, the background standard deviation (square root of background flux per pixel). The background is a function of time, as shown by Fig. 2; it can attain $30 \%$ of the stellar flux when the Sun is at its maximum, where typical values in ADU for the highest background are $B \simeq 1000$. It leads to a bias term $\sigma_{B}^{2} \simeq 10^{-4}$ square pixels, which is still negligible compared to the differential variance values.

\section{A.2. Isoplanatic angle}

Background noise. The presence of a strong background on individual images causes uncertainties and biases on the estimation of the mean stellar intensity $\bar{I}$ (ensemble average over the image sample), its standard deviation $\sigma_{I}$, and then on the scintillation index $s$. As shown in Fig. 2, the background can 
be as high as $30 \%$ of the stellar flux when the Sun is at its maximum. To perform a bias on SNR estimation, let us introduce the following variables:

- $B$, the background intensity collected over the $N_{I}$ pixels illuminated by the star after threshold application, $\bar{B}$, and $\sigma_{B}^{2}$ its mean and variance. $B$ is a Poisson random variable; so it must verify $\sigma_{B}=\sqrt{B}$, that was well verified on images.

- $I_{t}$ the total intensity (background+stellar flux) collected over the $N_{I}$ pixels.

The stellar flux is given by $I=I_{t}-B$, the measure being $I_{t}$. The mean $\bar{I}$ is biased by the term $\bar{B}$. This bias is estimated (we assume stationarity, so that the ensemble average $\bar{B}$ is equal to the average over one image) and removed as indicated above, but the background fluctuations lead to an error $\delta I$ on the estimation of $\bar{I}$ equal to $\delta I=\sigma_{B} \simeq \sqrt{B}$.

The variance $\sigma_{I}^{2}$ is equal to the difference $\sigma_{I}^{2}=\sigma_{I t}^{2}-\sigma_{B}^{2}$, assuming independence between the stellar flux and the background. The variance estimation we make on images is $\sigma_{I t}^{2}$; it is then biased by the term $\sigma_{B}^{2}$. However we noted that this bias is less than $1 \%$ of $\sigma_{I t}^{2}$ and decided not to debias the variances. In addition to this bias, there is an error term $\delta \sigma_{B}^{2}$ due to the uncertainty of the estimation of $\sigma_{B}^{2}$. Hence the total error on $\sigma_{I}^{2}$ is $\sigma_{B}^{2}+\delta \sigma_{B}^{2}$ if we do not debias the variances.

The scintillation index is the ratio $s=\sigma_{I}^{2} / \bar{I}^{2}$; its error $\delta s$ can be estimated by

$\frac{\delta s}{s}=\frac{\delta \sigma_{I}^{2}}{\sigma_{I}^{2}}+2 \frac{\delta \bar{I}}{\bar{I}}=\frac{\sigma_{B}^{2}+\delta \sigma_{B}^{2}}{\sigma_{I}^{2}}+2 \frac{\sigma_{B}}{\bar{I}}$.

Typical values corresponding to the worst case (strongest background at Sun's maximum elevation) are, in ADU units: $\sigma_{B} \simeq$ $400, \bar{I} \simeq 40000, \sigma_{I} \simeq 5000$ and $\delta \sigma_{B}^{2} \simeq 70000$. That gives a background error contribution $\frac{\delta s}{s}=3 \%$

Readout noise. The readout noise can be considered as a Gaussian random variable with mean $r$ (per pixel) and standard deviation $\sigma_{r}=2.3 \mathrm{ADU}$ (from Pixelfly documentation). As the star is spread over $N_{I}$ pixels, we will consider the variables $R=N_{I} r$ (mean over the $N_{I}$ pixels) and its standard deviation $\sigma_{R}=\sqrt{N_{I}} \sigma_{r}$. The mean value $R$ is automatically removed by the background substraction. The same reasoning as above can be applied to the readout noise. From Eq. (A.3) the contribution $\frac{\delta_{r} s}{s}$ of the readout noise is then given by

$\frac{\delta_{r} s}{s} \simeq \frac{\sigma_{R}^{2}}{\sigma_{I}^{2}}+2 \frac{\sigma_{R}}{\bar{I}}$ in which we have neglected the term $\delta \sigma_{R}^{2}$. Taking the same values as for the background noise, we have $\frac{\delta_{\mathrm{p}} s}{s} \simeq 10^{-3}$, which is one order of magnitude below the background noise and can be neglected.

\section{References}

Aristidi, E., Agabi, A., Vernin, J., et al. 2003a, A\&A, 406, L19

Aristidi, E., Agabi, A., Vernin, J., et al. 2003b, Mem. Soc. Astron. It. Suppl., 2, 146

Aristidi, E., Agabi, A., Azouit, M., et al. 2005, A\&A, 430, 739

Avila, R., Vernin, J., \& Cuevas, S. 1998, PASP, 110, 1106

Barletti, R., Ceppatelli, G., Paternò, L., Righini, A., \& Speroni, N. 1977, A\&A, 54, 649

Beckers, J. M., \& Zhong, L. 2002, BAAS, 34, 735

Borgnino, J., \& Brandt, P. N. 1982, JOSO Ann. Rep., 9

Brandt, P. N., Mauter, H. A., \& Smartt, R. 1987, A\&A, 188, 163

Coudé du Foresto, V., Swain, M., \& Schneider, J. 2004, Proc. Dome C A\&A, EAS eds., Toulouse, June 28-July 1st, 2004

Ehgamberdiev, S., Baijumanov, A., Ilyasov, S., et al. 2000, A\&AS, 145, 293

Fried, D. L. 1966, J. Opt. Soc. Am., 56, 1372

Frieden, B. R. 1983, Probability, statistical optics and data testing (Berlin: Springer), 248

Heintz, W. D. 1960, Veroff. Sternw. Munchen, 5, 100

Irbah, A., Laclare, F., Borgnino, J., \& Merlin, G. 1994, Sol. Phys., 149,213

Lawrence, J. 2004, Appl. Opt., 43, 1435

Lawrence, J., Ashley, M., Tokovinin, A. A., \& Travouillon, T. 2004, Nature, 431, 278

Loos, G., \& Hogge, C. 1979, Appl. Opt., 18, 2654

Marks, R., Vernin, J., Azouit, M., Manigault, J. F., \& Clevelin, C. 1999, A\&AS, 134, 161

Martin, H. M. 1987, PASP, 99, 1360

Martin, F., Conan, R., Tokovinin, A. A., et al. 2000, A\&AS, 144, 39

Muñoz-Tuñòn, C., Vernin, J., \& Varela, A. M. 1997, A\&AS, 125, 183

Pourbaix, D., Niveder, D., McCarthy, C., et al. 2002, A\&A, 386, 280

Ricort, G., \& Aime, C. 1979, A\&A, 76, 324

Roddier, F. 1981, Progress in optics XIX, 281

Sarazin, M., \& Roddier, F. 1990, A\&A, 227, 294

Tokovinin, A. 2002, PASP, 114, 1156

Tokovinin, A., Vernin, J., Ziad, A., \& Chun, M. 2005, PASP, 117, 395

Travouillon, T., Ashley, M., Burton, M., et al. 2003, A\&A, 400, 1163

Vernin, J., \& Muñoz-Tuñòn, C. 1995, PASP, 107, 265

Walters, D. L., Favier, D., \& Hines, J. R. 1979, J. Opt. Soc. Am., 69, 828

Ziad, A., Conan, R., Tokovinin, A., Martin, F., \& Borgnino, J. 2000, Appl. Opt., 39, 5415 